\title{
Relación entre el Bienestar Académico de Estudiantes de Kinesiología de una Universidad Tradicional de Chile y su Percepción del Ambiente Educacional
}

\author{
Camila P. Hinrichs, Liliana E. Ortiz yCristhian E. Pérez \\ Facultad de Medicina, Universidad de Concepción. Chacabuco esq. Janequeo, Barrio Universitario, \\ Concepción-Chile (e-mail: camihinrichs@gmail.com, lilianaortiz@udec.cl, cperezv@udec.cl)
}

Recibido Abr. 16, 2015; Aceptado Jun. 17, 2015; Versión final Ago. 17, 2015, Publicado Feb. 2016

\begin{abstract}
Resumen
Se ha analizado la relación entre percepción del ambiente educacional y bienestar académico. Se realizó un estudio exploratorio con una muestra de 163 estudiantes de Kinesiología. Previo consentimiento, a los se aplicó a los estudiantes una batería de cuestionarios para evaluar el compromiso académico (engagement académico), el síndrome de estar quemado académico (burnout académico) y la percepción del ambiente educacional. Para el análisis se usaron estadísticos descriptivos y coeficiente de correlación de Pearson. Se encontró correlación significativa en tres de cuatro factores de la escala de ambiente educacional con todos los factores de las escalas de compromiso académico y síndrome de estar quemado académico. Se concluye que una percepción más favorable del ambiente educacional se relaciona con mayores niveles de bienestar académico en estudiantes de Kinesiología. Es necesario planificar estrategias para mejorar la percepción de los docentes y disminuir el agotamiento emocional de los estudiantes.
\end{abstract}

Palabras clave: ambiente educacional; bienestar académico; síndrome de estar quemado académico; compromiso académico

\section{Relation between Academic Welfare of Physical Therapy Students of a Traditional University of Chile and their Perception of the Educational Environment}

\begin{abstract}
The relationship between the perception of students of the educational environment and academic welfare has been analyzed. An exploratory study was conducted with 163 students of physical therapy. With prior informed consent, a kit of questionnaires to assess their academic engagement, academic burnout and perception of the educational environment, were applied.For the analyses descriptive statisticsand Pearson's correlation coefficients were used.Significant correlation was found in three of the four factors of the educational environment scale with all of the factors of the academic engagement and burnout scales.It is concluded that a more favorable perception of the educational environment is associated with higher levels of academic welfare of physical therapist students. It is necessary to plan strategies to improve the perception of teachers and to reduce student'semotional exhaustion.
\end{abstract}

Keywords: educational environment; academic welfare; academic burnout; academic engagement 


\section{INTRODUCCIÓN}

La influencia del ambiente educacional sobre la satisfacción y éxito de los estudiantes ha sido reconocida en numerosas publicaciones (Hutchinson, 2003; Salanova et al., 2005; Herrera et al., 2010; Pérez et al., 2012; Ousey et al., 2014), correspondiendo a un indicador de calidad de la formación de pre y postgrado, asumido como un estándar mínimo por organizaciones de educación y agencias acreditadoras (Roff, 2005; Herrera et al., 2012; Llera y Durante, 2014). Se reconocen dos grupos de factores que se relacionan con el ambiente educacional: a) uno referido al plan de estudios, calidad de enseñanza, claridad de los procesos, resultados y mecanismos de apoyo, todos aspectos propios de la gestión curricular; b) y otro referido a estilos/técnicas de enseñanza, entusiasmo del docente y ambiente físico. La expresión virtuosa de estos factores podría promover un estado positivo de involucramiento y satisfacción con los estudios denominado "Engagement Académico" (Parra et al., 2010). En cambio, un ambiente educacional competitivo y con excesiva presión sobre los estudiantes podría generar un clima de tensión que afecte la motivación y el rendimiento (DíazVéliz, 2011; Riquelme et al., 2007). Desafortunadamente, la formación profesional en las carreras de la salud se caracteriza por un ambiente educacional marcado por una alta carga académica, modelos docentes inadecuados y agresiones desde los docentes y los alumnos, lo que conlleva a una expresión viciosa del ambiente educacional, (Pérez et al., 2012; Meyer et al., 2013; Pérez et al., 2013) la cual se ha relacionado con la presencia del "Síndrome de Burnout".

El Engagement académico, cuya traducción más literal al español sería compromiso, se define como un estado psicoafectivo positivo y persistente relacionado con los estudios, que se caracteriza por vigor o energía para estudiar y aprender, a pesar de los contratiempos y dificultades; dedicación e implicación en los estudios y carrera; y concentración y felicidad durante el desempeño de la actividad académica (Schaufeli, et al., 2002). Un alto nivel de Engagement académico indica un alto nivel de bienestar académico. Así, este constructo multidimensional ha sido asociado a mejores resultados académicos, por lo que también se debería asumir como un indicador del éxito de la gestión universitaria (Salanova et al., 2010; Parra, 2011). El problema es que lograr Engagement académico en los estudiantes es una tarea compleja, ya que el ingreso a la universidad puede convertirse en un momento crítico, al coincidir con la transición de la enseñanza media a la universidad y de la adolescencia a la adultez. Además, la educación superior enfrenta a los estudiantes a una forma de vivir que desconocen, a experiencias nuevas y a un momento en sus vidas en el que se ponen a prueba sus propias expectativas y las de su entorno, por lo que existe gran presión sobre ellos. En algunos casos, los estudios universitarios implican dejar el hogar, la familia y los amigos, lo que genera una pérdida de las redes de apoyo de los jóvenes y deja a los estudiantes en una situación de vulnerabilidad psicoafectiva (Beck, Taylor y Robbins, 2002; Dyson y Renk, 2006; Pérez et al., 2012).

En el lado opuesto al Engagement se encuentra el Síndrome de Burnout, también conocido como el síndrome de estar quemado, agotado o de desgaste, el cual se caracteriza por una actitud de pasividad, indiferencia, superficialidad y falta de colaboración de la persona en su trabajo o estudios. Es un síndrome negativo integrado por tres dimensiones: a) agotamiento emocional, que se caracteriza por falta o carencia de energía, de entusiasmo y un sentimiento de falta de recursos; b) despersonalización-cinismo, que se caracteriza por una respuesta impersonal, fría y cínica hacia los beneficiarios de los servicios o hacia los estudios; c) falta de realización personal-eficacia profesional, conducta evidenciada por una tendencia del sujeto a autoevaluarse en forma negativa, experimentando sentimiento de incompetencia y fracaso (Maslach y Jackson, 1986). Aunque este síndrome se describió en profesionales, también puede afectar a otras ocupaciones, como los estudiantes de los distintos niveles educativos, siendo los universitarios quienes estarían más expuestos a sufrir Burnout Académico (Backovic et al., 2012; Mazurkiewicz et al., 2012; Tomaschewski-Barlem et al., 2014).

Caballero et al (2010) consideran que vale la pena distinguir las investigaciones de este síndrome en alumnos de medicina y enfermería con los de otras carreras, dado que en esta población se retorna al concepto original de Burnout, el cual se visualiza en la relación estudiante-paciente. Además, las variables asociadas al síndrome se han identificado desde planteamientos teóricos y empíricos y podrían categorizarse en términos de su naturaleza en: a) variables del contexto académico; b) variables del contexto ambiental y/o social y c) variables intrapersonales (Caballero et al., 2010). El interés del presente estudio se encuentra en las variables académicas, por la responsabilidad que le corresponde a la universidad en formar profesionales que en el futuro estarán a cargo de la salud de la población. Las instituciones de educación superior no sólo deben procurar aprendizajes en los estudiantes y mantenerlos en el sistema, sino que también mejorar su experiencia educativa. Meda et al. (2012) lo explican en la siguiente frase: "La promoción de salud en el ámbito educativo constituye un valor agregado, al de por sí ya extraordinario valor que tiene la escuela". 
El objetivo del presente estudio es analizar la relación existente entre el ambiente educativo y el bienestar académico de los estudiantes de Kinesiología de una universidad tradicional de Chile, entendiendo la relevancia de estudiar este constructo en disciplinas sanitarias y ante la escasa literatura disponible en esta disciplina. Como hipótesis se plantea que al gestionar ambientes educativos satisfactorios se promueve el bienestar académico de los futuros profesionales de la salud, el que es un factor relevante de la salud física y mental de los estudiantes.

\section{METODOLOGÍA}

El presente estudio es de tipo cuantitativo no experimental, transversal y analítico relacional. La población corresponde a 186 estudiantes de kinesiología de una universidad tradicional de Chile de las cohortes 2010 a 2013. La muestra obtenida fue de 163 sujetos, elegidos por muestreo no probabilístico por accesibilidad, de los cuales 81 (49,69\%) eran mujeres y 82 (50,31\%) hombres, con edades entre los 19 y los 26 años $(\mathrm{M}=20,84 ; \mathrm{D} . \mathrm{E} .=1,38)$.

Para la recolección de datos se utilizaron las versiones validadas de 3 instrumentos: 1) la Escala Utrecht de Engagement ocupacional para estudiantes (Utrecht Work Engagement Scale for Students, UWES-17S) versión del cuestionario en español de 17 ítems agrupados en dos factores para medir el Engagement Académico (Schaufeli et al., 2002), el que ha mostrado evidencia de validez de constructo y criterio y de consistencia interna en un estudio de Parra (2011) realizado en estudiantes universitarios chilenos, 2) el Inventario de Burnout de Maslach-Encuesta de Estudiantes (Maslach Burnout Inventory-Student Survey, MBI-SS),versión de 22 ítems agrupados en tres factores para medir el Burnout Académico (Schaufeli et al., 2002), que cuenta con evidencia de su validez de constructo y consistencia interna en estudiantes universitarios de Chile (Pérez et al., 2013) y 3) la Escala de Dundee para evaluar Ambiente Educacional (Dundee Ready Educational Environment Measure, DREEM), versión de 50 ítems y cuatro factores para medir el Ambiente Educacional, cuya validez de constructo y consistencia interna ha sido apoya en un estudio de Ortega et al. (2015).

Para la aplicación de los instrumentos de recolección de datos se citó a los estudiantes y se les indicó a éstos los objetivos del estudio, en qué consistía éste, el tiempo que requeriría el contestar los cuestionarios y que su participación era absolutamente voluntaria. A los estudiantes que accedieron a participar se les solicitó que firmaran un consentimiento informado y se procedió a la aplicación de los instrumentos. Una vez terminada esta etapa de recolección de datos se dio paso al análisis de éstos.Para el análisis de los datos se evaluó la consistencia interna de los factores o subescalas de los instrumentos mediante coeficiente alfa de Cronbach, para estimar la precisión de las mediciones (Tavakol \& Dennick, 2011). Posteriormente, se realizó un análisis descriptivo de sus puntajes mediante media, desviación estándar, mínimo y máximo. Finalmente, se estableció la correlación entre las variables a través del coeficiente de correlación $r$ de Pearson en base a un contraste unilateral (Aron, Coups \& Aron, 2012), dado que se hipotetiza correlaciones directas entre las mediciones de Engagement, Burnout y Ambiente educacional (Llera \& Durante, 2014).

\section{RESULTADOS}

En primer lugar se presentan los estadísticos descriptivos del DREEM. De acuerdo a George y Mallery (2003), los valores del coeficiente alfa de Cronbach son pertinentes para los cuatro factores del DREEM, aunque cuestionables en los dos últimos casos. Asimismo, como no existe un baremos para la interpretación de este instrumento, se calculó el coeficiente M/i (media dividida por la cantidad de ítems)para facilitar la comparación entre factores. Dado que sus puntajes promedios están por sobre el punto medio posible de la escala DREEM, los estudiantes de kinesiología presentan niveles altos de "percepción académica", "percepción de la atmósfera y "experiencia académica"(M/i > 3),(Tabla1).

En general se percibe buena atmósfera durante las clases y actividades clínicas, así como oportunidades de desarrollo de habilidades interpersonales y comunicacionales. Además, existe una percepción positiva sobre las estrategias de enseñanza. Sin embargo, la valoración que hacen los estudiantes sobre la calidad, habilidades comunicacionales y de retroalimentación, nivel de conocimientos, preparación y planificación de las clases de sus profesores es inferior $(\mathrm{M} / \mathrm{i}<3)$.

Los valores del coeficiente alfa de Cronbach de las subescalas de UWES-17S resultaron aceptables para los dos factores. Respecto a los puntajes, el coeficiente M/i de las subescalas del UWES-17Sindican que los estudiantes de Kinesiología presentan niveles altos de "Involucramiento en los estudios" y "Satisfacción con los estudios"(M/i >4), (Tabla 2). Los valores del coeficiente alfa de Cronbach de las subescalas de MBI-SS fueron aceptables para los tres factores. Los puntajes de las subescalas del MBI SS indican que los estudiantes de Kinesiología presentan un nivel moderado de "Agotamiento emocional" y niveles bajos de "Despersonalización" y "Falta de realización personal" (M/i <4), (Tabla 3). Finalmente, para evaluar la 
correlación entre los puntajes del DREEM que reportan los estudiantes de Kinesiología y los puntajes de UWES-17S y MBI-SS, se calculó el coeficiente de correlación $r$ de Pearson en base a un contraste unilateral (Tabla 4).

Tabla 1. Consistencia interna y estadísticos descriptivos de DREEM en estudiantes de Kinesiología de una universidad tradicional, Chile. $\mathrm{N}=163$

\begin{tabular}{|l|l|l|l|l|l|l|}
\hline & $\alpha$ & $M$ & D.E. & Mín & Máx & M/i \\
\hline Percepción académica & 0,84 & 66,15 & 7,69 & 54 & 84 & 3,68 \\
\hline Experiencia académica & 0,74 & 31,57 & 5,29 & 13 & 44 & 3,51 \\
\hline Percepción de la atmósfera & 0,68 & 26,59 & 3,41 & 17 & 34 & 3,80 \\
\hline Percepción de los docentes & 0,66 & 12,38 & 3,42 & 11 & 28 & 2,06 \\
\hline
\end{tabular}

Tabla 2. Consistencia interna y estadísticos descriptivos de la UWES-17S en estudiantes de Kinesiología de una universidad tradicional, Chile. $\mathrm{N}=163$

\begin{tabular}{|l|l|l|l|l|l|l|}
\hline & $\alpha$ & M & D.E. & Mín & Máx & M/i \\
\hline Involucramiento en los estudios & 0,89 & 48,84 & 11,97 & 17 & 72 & 4,07 \\
\hline Satisfacción con los estudios & 0,84 & 25,10 & 5,36 & 3 & 30 & 5,02 \\
\hline
\end{tabular}

Tabla 3. Consistencia interna y estadísticos descriptivos del MBI-SS en estudiantes de Kinesiología de una universidad tradicional, Chile. $\mathrm{N}=163$

\begin{tabular}{|l|l|l|l|l|l|l|}
\hline & $\alpha$ & $M$ & D.E. & Mín & Máx & M/i \\
\hline Agotamiento emocional & 0,83 & 22,43 & 8,27 & 3 & 40 & 3,20 \\
\hline Despersonalización & 0,63 & 9,27 & 6,13 & 0 & 30 & 1,32 \\
\hline Falta de realización personal & 0,67 & 12,86 & 5,97 & 0 & 31 & 1,61 \\
\hline
\end{tabular}

Tabla 4. Correlación entre los puntajes de DREEM, UWES-17S y MBI-SSen los estudiantes de Kinesiología de una universidad tradicional, Chile. $N=163$; ${ }^{*} p<0,05$; ${ }^{*} p<0,01$; ${ }^{*} p<0,001$

\begin{tabular}{|c|c|c|c|c|c|c|c|c|c|}
\hline & 1 & 2 & 3 & 4 & 5 & 6 & 7 & 8 & 9 \\
\hline 1.Percepción académica & - & & & & & & & & \\
\hline 2. Experiencia académica & $0,57^{\star * *}$ & - & & & & & & & \\
\hline 3.Percepción de la atmósfera & $0,56^{\star * *}$ & $0,39^{\star * *}$ & - & & & & & & \\
\hline 4.Percepción de los docentes & $0,31^{* * *}$ & $0,15^{*}$ & $0,44^{\star \star \star}$ & - & & & & & \\
\hline 5. Involucramiento en los estudios & $0,47^{\star * *}$ & $0,51^{* * *}$ & $0,27^{\star \star \star}$ & $0,18^{\star *}$ & - & & & & \\
\hline 6. Satisfacción con los estudios & $0,43^{\star * *}$ & $0,33^{* * *}$ & $0,14^{*}$ & 0,11 & $0,61^{\star * *}$ & - & & & \\
\hline 7. Agotamiento emocional & $-0,24^{\star \star *}$ & $-0,37^{* * *}$ & $-0,19^{* *}$ & $-0,12$ & $-0,01$ & 0,10 & - & & \\
\hline 8. Despersonalización & $-0,22^{* *}$ & $-0,16^{*}$ & $-0,17^{*}$ & $-0,15^{*}$ & 0,07 & $<0,01$ & $0,21^{* *}$ & - & \\
\hline 9. Falta de realización personal & $-0,41^{* * *}$ & $-0,41^{* * *}$ & $-0,24^{* *}$ & 0,01 & $-0,40^{* * *}$ & $-0,38^{* * *}$ & 0,06 & $0,22^{* *}$ & - \\
\hline
\end{tabular}

De acuerdo a la correlación existente entre la percepción del ambiente educativo que reportan los estudiantes de Kinesiología y sus niveles de bienestar académico, se puede observar que tres de los cuatro factores de la escala DREEM correlacionan con todos los factores del MBI-SS y UWES-17S.

\section{DISCUSIÓN}

Con respecto a la percepción del clima académico por parte de los estudiantes de kinesiología de una universidad tradicional de Chile, éstos tienen una percepción académica y de la atmósfera y una experiencia académica más bien positiva, lo que indica que existe una buena atmósfera durante las clases y pasantías en centros clínicos, buenas oportunidades de desarrollo de habilidades interpersonales y comunicacionales y motivación por parte de los docentes mediante sus actividades. Además, se evidencia que los estudiantes 
tienen una percepción positiva sobre sus estrategias de aprendizaje y sus habilidades para resolver problemas. Ahora bien, de acuerdo a la percepción de los estudiantes con respecto a los docentes, ésta es más negativa, lo que indica que el alumnado tiene una peorvisión con respecto a la calidad de los docentes, sus habilidades comunicacionales y de retroalimentación, su nivel de conocimientos, preparación y planificación de las clases.

Los resultados de las escalas de Engagement y Burnout académico muestran que los estudiantes se encuentran satisfechos y comprometidos con sus estudios, pero presentan un nivel moderado de agotamiento emocional. Considerando que los estudiantes de kinesiología deben trabajar con personas, esto concordaría con el estudio realizado por Agut et al., (2002), quienes demostraron que los estudiantes de ciencias humanas pueden presentar un mayor agotamiento emocional que el que presentan estudiantes de otras disciplinas. Esto también se asociaría a lo encontrado por Formighieri (2003), que apunta a varios factores estresantes presentes en la actuación de los kinesiólogos y sus estudiantes que pueden llevar al agotamiento, siendo la expectativa de rehabilitación de los pacientes uno de los principales. Este mismo autor identificó niveles altos de agotamiento emocional y niveles moderados de despersonalización y realización personal en un estudio en kinesiólogos, lo que representa la fase inicial del síndrome de Burnout. A partir de esto es importante señalar que algunas dimensiones del síndrome de Burnout pueden comenzar a gestarse durante la vida universitaria de los estudiantes de kinesiología, mantenerse e incluso empeorar una vez que éstos ya se enfrentan a la vida profesional. Por ello, la prevención del Burnout desde el período de formación es importante, pues los profesionales del área de la salud, debido a que prestan atención directa a otras personas, están constantemente sometidos a una amplia variedad de fuentes de estrés, por lo que pueden ser considerados como un grupo particularmente vulnerable (Gil-Monte, 2002).

A partir de la relación encontrada entre las escalas de ambiente educacional y las de Engagement y Burnout académico, es importante destacar cómo la experiencia de los estudiantes y la percepción que éstos tienen del ambiente educacional condicionan su bienestar en todos los niveles de la carrera, lo que concuerda con lo mencionado por Díaz-Véliz (2011), quien concluyó que la percepción que los estudiantes tienen del ambiente donde estudian puede ejercer un impacto significativo sobre la efectividad del aprendizaje, el progreso académico y la sensación de bienestar. El único factor de la escala DREEM que no se correlaciona con todos los factores del Burnout y el Engagement es el de percepción de los docentes, donde éste no condiciona la satisfacción con los estudios, el agotamiento emocional ni la falta de realización, lo que permite suponer que la opinión que los estudiantes poseen de sus académicos tiene menor relación con el bienestar académico percibido.

La percepción de los docentes sólo correlaciona directamente con el Involucramiento en los estudios y de forma inversa con la Despersonalización. Esto muestra que el grado en que los estudiantes validan a la planta docente puede influir directamente en el nivel de esfuerzo que estos están dispuestos a entregar en sus actividades académicas, aspecto que tiene una influencia importante en el desempeño académico de los estudiantes. Por otro lado, es interesante destacar que la correlación inversa entre despersonalización y percepción de los docentes podría deberse a que los docentes pueden llegar a perturbar la forma de establecer relaciones y empatizar de los estudiantes. En casos de ausencia de relaciones empáticas y retroalimentación positiva, los docentes se transformarían en modelos de "cinismo y despersonalización" (Carlotto, 2005).Ahora bien, en relación a la percepción que los estudiantes tienen de las actividades académicas (subescala de Percepción académica), se puede observar que ésta tiene una correlación directa y estadísticamente significativa con los factores de la escala de Engagement e inversa con los factores de la escala de Burnout. Esto muestra que mientras mejor evaluados son los procesos formativos al que los estudiantes son expuestos, mayores son los niveles de bienestar académico, evidenciado por un mayor compromiso y menor Burnout.

\section{CONCLUSIONES}

De los resultados mostrados, de su análisis y de su discusión se pueden obtener las siguientes conclusiones sobre la percepción del ambiente educacional y su relación con el bienestar académico en estudiantes de Kinesiología de una universidad tradicional chilena: 1) la percepción general del ambiente educacionales positiva,2) la percepción de los docentes es más negativa, 3) los estudiantes presentan altos niveles de involucramiento y satisfacción con los estudios, pero también presentan alto nivel de agotamiento emocional, 4)la percepción del ambiente educacional se relaciona con los niveles de bienestar académico en estudiantes de Kinesiología, 5) los estudiantes con agotamiento emocional presentan peor percepción del ambiente educacional y menor involucramiento con los estudios, 6) el comité de currículum deberá planificar estrategias para mejorar la percepción de los docentes y disminuir el agotamiento emocional de los estudiantes. 


\section{AGRADECIMIENTOS}

Proyecto financiado por Conicyt a través del Proyecto FONDECYT № 1121002 "Proceso de adaptación a la universidad de los estudiantes de medicina durante los primeros tres años de su carrera y su relación con el perfil sociodemográfico del alumno y las características del entorno académico"

\section{REFERENCIAS}

Agut, S., Grau, R. y Beas, M., Burnout em mujeres: umestudo comparativo entre contextos de trabalho e no trabalho. [On-line]. V Congreso Galaico-Português de Psicopedagogía. IX Congreso de la Sociedade española de Psicología. III Jornada de la Sociedad Portuguesa de Psicología. Disponible en: http:// fsmorente.filos.ucm.es/publicaciones/ Iberpsicologia/congreso/programa.htm (2002)

Aron, A., Coups,E. y Aron, E, Statistics for Psychology 6th Edition. Pearson: New Yersey (2012)

Beck, R., Taylor, C. y Robbins, M, Missing home: sociotropy and autonomy and their relationship to psychological distress and homesickness in college freshmen, Anxiety, stress and coping. 16 (2), 155-166 (2002)

Backovic, D., llić Živojinović, J., Maksimović, J. y Maksimović, M, Gender differences in academic stress and burnout among medical students in final years of education, Psychiatria Danubina, 24(2), 175-181 (2012)

Caballero, C., Hederich, C. y Palacio, J., El burnout académico: delimitación del síndrome y factores asociados con su aparición, Revista Latinoamericana de Psicología, 42(1), 131-146 (2010)

Carlotto M., Goncalves S. y Brazil A., Predictores del síndrome de burnout en estudiantes de un curso técnico de enfermería, Diversitas: Perpectivas en psicología, 2(1), 195-205 (2005)

Díaz-Véliz G., Percepción del ambiente educativo en una facultad con currículo tradicional y otra con currículo basado en problemas. EducMed, 14(1), 27-34 (2011)

Dyson, R. y Renk, K, Freshmen adaptation to university life: depressive symptoms, stress, and coping, Journal of clinical psychology, 62 (10), 1231-1244 (2006)

Formighieri V., Burnout em fisioterapeutas: influencia sobre a atividade de trabalho ebem-estar físico e psicológico, Tesis de magíster,Universidade Federal de Santa Catarina, Florianópolis , Brasil (2003)

George, D. y Mallery, P. SPSS for Windows step by step: A simple guide and reference, $4^{\underline{a}}$ edición. Allyn \& Bacon, Boston, Estados Unidos (2003)

Gil-Monte P., Influencia del género sobre el proceso de desarrollo del síndrome de quemarse por el trabajo (Burnout) en profesionales de enfermería, Psicología em Estudo, 7(1), 3-10 (2002)

Herrera, C., Pacheco, J., Rosso, F., Cisterna, C., Aichele, D. Becker, S., Evaluación del ambiente educacional pre-clínico en seis Escuelas de Medicina en Chile, Rev. méd. Chile, 138 (6), 677-684 (2010)

Herrera, C. y otros 10 autores. Evaluación del ambiente educacional en programas de especialización médica, Rev. méd. Chile, 140(12), 1554-1561 (2012)

Hutchinson, L., Educational environment, BMJ, 326 (7393), 810-812 (2003)

Llera, J. y Durante, E., Correlación entre el clima educacional y el síndrome de desgaste profesional en los programas de residencia de un hospital universitario, Arch Argent Pediatría: 112(1), 6-11 (2014)

Maslach, C. y Jackson, S. Maslach Burnout Inventory Manual. 2nd ed.: Palo Alto (1986)

Mazurkiewicz, R., Korenstein, D., Fallar, R. y Ripp, J, The prevalence and correlations of medical student burnout in the pre.clinical years: A cross-sectional study, Psychology, Health and Medicine, 17(2), 188-195 (2012)

Meda, R., De Santos, F., Palomera, A. y Del Toro, R., Educar para la salud en Educación Superior: Tendencias y retos, RevEducCienc Salud, 9 (2), 86-94 (2012)

Meyer, A., Ramírez, L. yPérez, C., Percepción de estrés en estudiantes chilenos de Medicina y Enfermería, Revista de Educación en Ciencias de la Salud, 10 (2), 79-85 (2013) 
Ortega, J. y otros 8 autores. Estructura factorial de la escala DREEM en estudiantes de Medicina Chilenos, Rev.méd. Chile, 143, 651-657 (2015)

Ousey, K., Stephenson, J., Brown, T. y Garside, J, Investigating perceptions of the academic educational environment across six undergraduate health care courses in the United Kingdom, Nurse Education in Practice, 14, 24-29 (2014)

Parra, P. y Pérez, C., Propiedades psicométricas de la escala de compromiso académico, UWES-S (versión abreviada), en estudiantes de psicología, RevEducCienc Salud, 7(2), 128-133 (2010)

Parra, P., Efecto del engagement académico sobre el rendimientoteórico y práctico, tesis de Magister, Departamento de Educación Médica, Universidad de Concepción, Chile (2011)

Pérez, C y otros 6 autores, Problemas de salud mental en estudiantes universitarios de primer año de Concepción, Chile, Anales de Psicología: 28(3), 797-804 (2012)

Pérez, C., Maldonado, P., Aguilar, C. y Acosta, M., Ambiente Educacional y su relación con la salud mental de estudiantes universitarios chileno, Revista Argentina de Clínica Psicológica: 21(3), 257-268 (2013)

Riquelme, A., Fuentes, G., Jeria, A., Méndez, I., Aranís, C., Larios, G. y Oporto, J., Ambiente educacional y calidad de la docencia en la escuela de medicina, ARS méd: 15(15), (2007)

Roff, S., The Dundee Ready Educational Environment Measure (DREEM)-a generic instrument for measuring students' perceptions of undergraduate health professions curricula, Med Teach: 27(4), 322-5 (2005)

Salanova, M., Martínez, I., Bresó, E., Llorens, S. y Grau, R., Bienestar psicológico en estudiantes universitarios: facilitadores y obstaculizadores del desempeño académico, Anales de Psicología, 21(01), 170-180 (2005)

Salanova, M., Schaufeli, W., Martínez, I y Bresó, E., How obstacles and facilitators predict academic performance: the mediating role of study burnout and engagement, Anxiety, Stress \& Coping, 23(1), 53-70 (2010)

Schaufeli, W.B., Martínez, I.M., Marques, A., Salanova, M., Bakker, A.B., Burnout and engagement in university students: A crossnational study, Journal of Cross-Cultural Psychology, 33(5), 464-481 (2002)

Schaufeli, W.B., Salanova, M., González-Romá, V., Bakker, A. The measurement of Engagement and Burnout: A two sample confirmatory factor analytic approach, Journal of Happiness Studies, 3, 71-92 (2002)

Tavakol, M. y Dennick, R, Making sense of Cronbach's alpha, International journal of medical education, 2, $53(2011)$

Tomaschewski-Barlem, J. et al. Burnout syndrome among undergraduate nursing students at a public university, Rev. Latino-Am. Enfermagem, Ribeirão Preto, 22(6), 934-941 (2014) 
